\title{
Could Edge-Lit Type Ia Supernovae be Standard Candles?
}

\author{
Enikő Regös ${ }^{1}$ Christopher A. Tout \\ Institute of Astronomy, The Observatories, Madingley Road, Cambridge CB3 OHA \\ Dayal Wickramasinghe \\ Astrophysical Theory Centre, Australian National University, Canberra, ACT \\ 0200, Australia \\ Jarrod R. Hurley \\ American Museum of Natural History, Central Park West at 79th Street, New \\ York, U.S.A. \\ Onno R. Pols ${ }^{2}$ \\ Department of Mathematics, Monash University, Clayton, Victoria 3168, \\ Australia
}

\begin{abstract}
The progenitors of Type Ia supernovae (SNe Ia) have not been identified. Though they are no longer fashionable we investigate the consequences if a significant number of SNe Ia were edge-lit detonations (ELDs) of carbon/oxygen white dwarfs that have accreted a critical mass of helium. Our best understanding of the Phillips relation between light curve speed and peak luminosity assigns both these phenomena to the amount of ${ }^{56} \mathrm{Ni}$ produced. In ELDs there are two sites of ${ }^{56} \mathrm{Ni}$ synthesis. If the peak luminosity is determined primarily by the $\mathrm{CO}$ ratio in the core it is primarily a function of the initial main-sequence mass of the progenitor of the $\mathrm{CO}$ white dwarf. If the light curve decay speed is determined by the total mass of iron group elements ejected this is a function of the total mass of the ELD at the time of explosion because both the $\mathrm{CO}$ core and the He envelope are substantially converted to ${ }^{56} \mathrm{Ni}$. In general, binary star evolution ensures that these two masses are correlated and an empirical relation between peak luminosity and light curve shape can be expected. However when we perform population synthesis for progenitors of different metallicities we find a systematic shift in this relation that would make distant ELD SNe Ia fainter than those nearby. The abundances of alpha-rich isotopes, such as ${ }^{44} \mathrm{Ca}$, in the solar system indicate that only about 40 per cent of SNe Ia could be edge-lit so any systematic effect that could be present would be correspondingly diluted. A
\end{abstract}


systematic effect is still expected even if we examine only the small subset of ELDs that accrete from a naked helium star, rather than a He white dwarf.

Key words: stars: evolution, binaries: close, supernovae: general, cosmology: observations, distance scale

PACS: 97.60, 97.60.B, 97.80

\section{Introduction}

Luminous type Ia supernovae (hereinafter SNe Ia) are amongst the brightest objects in the Universe. Observations indicate that their absolute magnitudes lie in a narrow range, $M_{\mathrm{B}}=-18.5 \pm 0.5$, or \pm 60 per cent in luminosity (from Table 1 of Rowan-Robinson 2002). Subluminous objects such as SN1991bg (Filippenko et al. 1992) lie below this range but have quite different spectral features. Furthermore observations reveal a correlation between maximum absolute brightness and light curve shape that facilitates an effective reduction in the standard deviation of absolute luminosities of all SNe Ia to \pm 15 per cent (Phillips 1993, Phillips et al. 1999). This small spread, coupled with the fact that they can be seen to great distances makes SNe Ia excellent standard candles for study of the cosmology of the Universe. Observations have determined the rate of expansion with unprecedented precision (Branch 1998) and have further determined that this rate is accelerating with time, a measurement that has led to the invocation of a cosmological constant contributing about 70 per cent of the critical density of the Universe to be added to matter's contribution of 30 per cent (Perlmutter et al. 1999, Riess et al. 1998). Further credence is lent to this result by cold-dark-matter models of the angular variation of the cosmic microwave background (de Bernardis et al. 2000) and gravitational lensing measurements (Wittman et al. 2000). Taken together, these results strongly favour a universe dominated by a new form of energy, as yet unexplained.

Each of the steps leading to the above conclusions needs to be critically analysed. In this paper we look at the assertion that SNe Ia can be used as standard candles in the light of what we know from current state-of-the-art binary population synthesis calculations. These calculations point to a range of possibilities for the progenitors of SNe Ia, none of which can be eliminated with any certainty. Likely candidates are double-degenerate super-Chandrasekhar

1 E-mail: eniko@ast.cam.ac.uk

2 Present Address: Astronomical Institute Utrecht, Utrecht University, P.O. Box 80000, 3508 TA Utrecht, Netherlands 
white dwarf mergers, single-star Chandrasekhar-mass CO white dwarf ignitors, and edge-lit detonations (ELDs) of carbon/oxygen white dwarfs that have accreted a critical mass of helium. The relative populations predicted for these types are model dependent, although our calculations (Hurley, Tout \& Pols 2001) predict a dominant population of ELDs. Though only a few attempts have been made to calculate spectra of edge-lit detonations (Höflich and Khokhlov 1996, Nugent et al. 1997) these find that the synthetic light curves do not match the observations as well as those for the standard model of carbon ignitors and that the spectra are too blue. However, the possibility that the ELDs may be a significant contributor to SNe Ia cannot be ruled out on these grounds at present because a comprehensive set of explosion calculations are presently unavailable (Branch 2000) and alternatives may indeed be found to fit the data.

In this paper we point out that, if a significant fraction of SNe Ia were ELDs, there is a systematic effect that can be identified which could make the more distant SNe Ia fainter than the nearby. The evidence for an accelerating universe from SNe Ia, which requires they be standard candles, would then be undermined. The abundance of alpha rich isotopes such as ${ }^{44} \mathrm{Ca}$ in the solar system suggests that perhaps 40 per cent of SNe Ia could be ELDs.

\section{Binary Star Progenitors}

The major energy source of SNe Ia is the decay of ${ }^{56} \mathrm{Ni}$ to ${ }^{56} \mathrm{Fe}$ and the total energy released in a SN Ia is consistent with the decay of approximately a solar mass of ${ }^{56} \mathrm{Ni}$. These facts strongly implicate the thermonuclear explosion of a white dwarf though the actual explosion mechanism is not fully understood (Hillebrandt \& Niemeyer 2000). White dwarfs may be divided into three major types: (i) helium white dwarfs, composed almost entirely of helium, form as the degenerate cores of low-mass red giants $\left(M \leq 2 M_{\odot}\right)$ which lose their hydrogen envelope before helium can ignite; (ii) carbon/oxygen white dwarfs, composed of about 20 per cent carbon and 80 per cent oxygen, form as the cores of asymptotic giant branch stars or naked helium burning stars that lose their envelopes before carbon ignition (with progenitors typically in the range $1-6 M_{\odot}$ ); and (iii) oxygen/neon white dwarfs, composed of heavier combinations of elements, form from giants that ignite carbon in their cores but still lose their envelopes before the degenerate centre collapses to a neutron star (with progenitors typically in the range $6-8 M_{\odot}$ ).

An overview of the evolution of close binary systems is given by Hurley, Tout $\&$ Pols (2002; and references therein) and we review here those aspects which are directly relevant to SNe Ia. In such systems, mass transfer can increase the mass of a white dwarf. Close to the Chandrasekhar mass $\left(M_{\mathrm{Ch}} \approx 1.44 M_{\odot}\right)$ 
degeneracy pressure can no longer support the star which collapses releasing its gravitational energy. The ONe white dwarfs lose enough energy in neutrinos and collapse sufficiently, before oxygen ignites, to avoid explosion (accretion induced collapse, AIC). The CO white dwarfs, on the other hand, reach temperatures early enough during collapse for carbon fusion to set off a thermonuclear runaway under degenerate conditions and release enough energy to create a SN Ia. Accreting He white dwarfs reach sufficiently high temperatures to ignite helium well below $M_{\mathrm{Ch}}\left(M \approx 0.7 M_{\odot}\right.$, Woosley, Taam \& Weaver 1986) but an explosion under these conditions is expected to be quite unlike a SN Ia.

The process is further complicated by the nature of the accreting material. If it is hydrogen-rich, accumulation of a layer of only $10^{-4} M_{\odot}$ or so leads to ignition of hydrogen burning sufficiently violent to eject most, if not all of or more than, the accreted layer in the well known novae outbursts of cataclysmic variables (Warner 1995). The white dwarf mass does not significantly increase and ignition of its interior is avoided. However if the accretion rate is high $\dot{M}>10^{-7} M_{\odot} \mathrm{yr}^{-1}$ hydrogen can burn as it is accreted, bypassing novae explosions (Paczyński \& Żytkow 1978), and allowing the white dwarf mass to grow. Though, if it is not much larger than this, $\dot{M}>3 \times 10^{-7} M_{\odot} \mathrm{yr}^{-1}$, hydrogen cannot burn fast enough so that accreted material builds up a giant-like envelope around the core and burning shell which rapidly leads to more drastic interaction with the companion and the end of the mass transfer episode. Rates in the narrow range for steady burning are found only when the companion is in the short-lived phase of thermal-timescale expansion as it evolves from the end of the main sequence to the base of the giant branch. Super-soft X-ray sources (Kahabka \& van den Heuvel 1997) are probably in such a state but, without invoking a special feedback mechanism (Hachisu, Kato \& Nomoto 1996), cannot be expected to remain in it for very long and white dwarf masses very rarely increase sufficiently to explode as SNe Ia. This however remains the currently favoured progenitor, though we note that the continuing absence of a detection of hydrogen that may have been stripped from the mass-losing companion, either at early stages (Cumming et al. 1996) or late (Eck et al. 1995), may change this popular opinion in the future.

At first sight, a more promising scenario might be mass transfer from one white dwarf to another. In a very close binary orbit gravitational radiation can drive two white dwarfs together until the less massive fills its Roche lobe. If both white dwarfs are CO and their combined mass exceeds $M_{\mathrm{Ch}}$ enough mass could be transferred to set off a SN Ia. However if the mass ratio $M_{\text {donor }} / M_{\text {accretor }}$ exceeds 0.628 mass transfer is dynamically unstable because a white dwarf expands as it loses mass. Based on calculations at somewhat lower, steady accretion rates, Nomoto \& Iben (1985) have claimed that the ensuing rapid accretion of material allows carbon to burn in mild shell flashes, converts the white dwarf to ONe and ultimately leads to AIC and not a SN Ia. For smaller 
mass ratios accretion proceeds on the gravitational radiation timescale and in some cases $M_{\mathrm{Ch}}$ can be reached.

If a $\mathrm{CO}$ white dwarf accretes from a He white dwarf the mass ratio is generally small enough for dynamically stable mass transfer so that a helium layer builds up on the surface of the $\mathrm{CO}$ white dwarf. As in the nova explosions of hydrogen, the base of this helium layer eventually reaches a temperature at which helium can ignite in a degenerate flash. Unlike the novae this requires about $0.15 M_{\odot}$ of helium (Woosley \& Weaver 1994). It can be envisaged that ignition of such a massive helium layer can detonate the $\mathrm{CO}$ core either by compressing it or by an inwardly propagating heating front (Branch \& Nomoto 1986; Livne \& Glasner 1990). Typical total masses of these edge-lit detonations (hereinafter ELDs), though below $M_{\mathrm{Ch}}$, are still of the order of a solar mass so enough energy exists to explode the star and enough ${ }^{56} \mathrm{Ni}$ can be formed to make a SN Ia.

Our population synthesis predicts, in general, more ELDs than merging CO white dwarfs by typically a factor of six (twenty-eight if only dynamically stable mass transfer is counted). Note that Kawai, Saio \& Nomoto (1987) investigated helium accreting spherically on to a CO core and claimed that helium can burn non-degenerately if accreted steadily at rates above about $3 \times$ $10^{-8} M_{\odot} \mathrm{yr}^{-1}$ and so avoid setting off an ELD. Because gravitational radiation drives mass transfer from a helium white dwarf donor at a rate considerably in excess of this, progenitors involving two white dwarfs have been ruled out in the past. However, if accretion is through a disc the $\mathrm{CO}$ core is able to cool as it accretes and a degenerate layer of helium can still build up.

Spectral analysis of SNe Ia has also been inconclusive. The spectra of the very luminous SN Ia $1991 \mathrm{~T}$ are consistent with ${ }^{56} \mathrm{Ni}$ production both at its centre and in a shell at the outside (Liu, Jeffery \& Schultz 1997, Fisher et al. 1999). Though Fisher et al. (1999) favour a super-Chandrasekhar mass model, this is consistent with the two sites of thermonuclear runaway, the helium envelope and the $\mathrm{CO}$ core, present in an ELD. A potential problem with any claim that the majority of SNe Ia are ELDs is the lack of helium found in their spectra (Mazzali \& Lucy 1998). However there remains sufficient uncertainty in the explosion models, such as exactly how much helium survives the explosion (typically less than $0.08 M_{\odot}$ in the calculations of Livne \& Arnett, 1995), and the conversion to observed spectra, in particular the assumption that the helium shell remains spherically symmetric around the exploding CO core, that they cannot be ruled out with certainty. This problem is no more severe than is the lack of evidence for hydrogen for the currently favoured single degenerate models. Indeed ELDs have received almost no attention in recent years and it may be worthwhile to revisit them in the light of improved modelling applied to the standard model. 
Table 1

Supernovae rates for various progenitors per $1,000 \mathrm{yr}$ and their yields.

$\begin{array}{lccccc} & \alpha_{\mathrm{CE}}=1 & \alpha_{\mathrm{CE}}=3 & M_{\mathrm{Ni}} / M_{\odot} & M_{\mathrm{Ti}} / M_{\odot} & M_{\mathrm{Ca}} / M_{\mathrm{Fe}} \\ & & & & & \\ \text { Coalescence CO + CO } & 0.05 & 1.20 & 0.76 & 2.2 \times 10^{-6} & 1.9 \times 10^{-4} \\ \text { CO on to CO accretion } & 0.04 & 0.36 & 0.23 & 6.6 \times 10^{-7} & 1.9 \times 10^{-4} \\ & & & & & \\ \text { CO + He wd ELD } & 2.90 & 8.79 & 5.07 & 3.6 \times 10^{-2} & 5.1 \times 10^{-3} \\ \text { CO + naked He ELD } & 0.36 & 1.12 & 0.71 & 4.3 \times 10^{-3} & 4.6 \times 10^{-3} \\ & & & & & \\ \text { He wd + He wd ignition } & 0.15 & 1.39 & 0.63 & 1.2 \times 10^{-2} & \left(1.3 \times 10^{-2}\right) \\ \text { He wd + naked He ignition } & 0.00 & 0.28 & 0.13 & 2.5 \times 10^{-3} & \left(1.3 \times 10^{-2}\right) \\ & & & & & \\ \text { Total CO > } M_{\text {Ch }} & 0.09 & 1.57 & 0.99 & 2.8 \times 10^{-6} & 1.9 \times 10^{-4} \\ \text { Total ELD } & 3.26 & 9.91 & 5.79 & 4.0 \times 10^{-2} & 5.0 \times 10^{-3} \\ \text { Total He wd ignition } & 0.15 & 1.67 & 0.75 & 1.5 \times 10^{-2} & \left(1.3 \times 10^{-2}\right) \\ & & & & & \\ \text { Total } & 3.50 & 13.15 & 7.52 & 5.5 \times 10^{-2} & 5.3 \times 10^{-3}\end{array}$

\section{Population Synthesis}

We might hope to gain some insight into the relative sizes of the progenitor populations from binary population synthesis calculations. For example we list results from calculations of this type with the algorithms described by Hurley, Tout \& Pols (2002) in Table 1. The parameter $\alpha_{\mathrm{CE}}$ that measures the efficiency of the common envelope ejection is one of the most critical and least constrained. In order to find enough merging $\mathrm{CO}$ white dwarfs to approach the measured rate of $4 \pm 2$ SNe Ia per 1,000 yr per galaxy like our own (Cappellaro et al. 1997) requires $\alpha_{\mathrm{CE}} \geq 3$. In this case only about 20 per cent of merging white dwarfs avoid dynamically unstable mass transfer and these may be too rare to account for the SNe Ia rate observed locally. However the almost unlimited freedom to vary such physical parameters as $\alpha_{\mathrm{CE}}$ together with all initial distributions of binary parameters (binary fraction, mass ratio, period etc.) convinces us that we cannot trust such absolute numbers from population synthesis.

Given an initial state for a binary system, its masses $M_{1}$ and $M_{2}$ and its pe- 
riod $^{3} P$, and a model for all the physical processes of stellar evolution and binary interaction, we can determine whether or not a particular system would end its life as a SN Ia. These physical processes include mass transfer both by Roche-lobe overflow or in a stellar wind, common-envelope evolution, magnetic and gravitational-radiation braking and all the associated effects on the evolution of the individual components that comprise the system. Unfortunately many of them are not sufficiently well understood for a precise quantification. Particularly troublesome for SNe Ia is common-envelope evolution because it is necessary to bring white dwarfs close enough to interact.

When a red giant or AGB star grows to fill its last stable potential surface or Roche lobe it begins to transfer mass to its companion. But, as it loses mass, the convective envelope of a giant expands. If it is still the more massive component of the binary, and mass transfer is conservative, the orbit and Roche lobe shrink. Consequently the process of mass transfer leads, on a dynamical timescale, to the giant overfilling its Roche lobe yet more. The overflow rate rapidly rises and the companion, typically a lower-mass main-sequence star, cannot accrete the material. Its own Roche lobe is quickly filled and a common envelope engulfs the whole system. The two cores, the relatively dense companion and the core of the giant are then assumed to spiral together by some, as yet undetermined, frictional mechanism. Some fraction of the orbital energy released is available to drive off the envelope. If all of it is ejected while the cores are still well separated we are left with a closer binary system comprising the unscathed companion and a white dwarf which may evolve to a cataclysmic variable. Alternatively, if some of the envelope still remains when the companion reaches the denser depths of the common envelope, they can merge leaving a single, rapidly rotating giant. Magnetic braking quickly spins down these merged giants.

Our ignorance of this process is encapsulated in a constant $\alpha_{\mathrm{CE}}$ which measures the fraction of the released orbital energy that goes into driving off the envelope. Very few agree on the precise definition of $\alpha_{\mathrm{CE}}$ and its numerical value is uncertain to within a factor of ten. Because additional energy is always available from the thermal reservoir provided by the nuclear burning luminosity it may well exceed unity. Furthermore it is almost certainly not constant from one case to another (Regös \& Tout 1995).

Any white dwarf accreting matter from its companion must have passed through at least one phase of common-envelope evolution because the white dwarf must itself have been the core of a giant that could only have evolved in

3 In practice a binary star may have an initial eccentricity but, in general, tides circularise the orbit before significant interaction takes place. Because angular momentum is conserved during circularisation it is actually the distribution of semi-latera recta that is appropriate (Hurley, Tout \& Pols 2002). 
a much wider system. Many double degenerate systems have passed through two phases. We divide the exploding white dwarfs into three types, (i) exploding Chandrasekhar-mass CO white dwarfs, (ii) ELDs and (iii) exploding He white dwarfs. Each type separates into groups of low and high intial-period systems. The high-period systems experience two common-envelope phases. The first, when the primary fills its Roche lobe as a giant, leaves the binary wide enough for the secondary also to evolve to a giant before it too fills its Roche lobe. The low-period systems experience only the second phase. Mass transfer from the primary begins while it is crossing the Hertzsprung gap, or perhaps while it is still on the main sequence. Mass transfer then proceeds only on a thermal timescale. After this Algol phase of evolution we are left with a white dwarf companion to a more massive secondary in a much wider system. The secondary then evolves to fill its Roche lobe only as a giant. Most of the progenitors fall into one of these two categories but some follow a considerably more convoluted evolution. Although we are fully aware of the limitations of population synthesis we believe our model to be better than any used by others because of its fuller treatment of tidal interaction and more careful modelling of Hertzsprung gap evolution and stellar-wind mass transfer.

A lack of understanding of common-envelope evolution alone ought to be enough to make us very wary of the results of population synthesis. But, like others before us, we go further and calculate the SNe Ia rate for the various progenitors in a typical galaxy like our own. To do this we convolve a grid of models (about 5,000,000 are needed to sufficiently resolve the $M_{1}-M_{2}-P$ space) with initial mass functions for each of the components, an initial period distribution, a binary fraction, a star formation history and a galactic model. Although each of these might be reasonably guessed on its own, together they give us enough freedom to achieve almost any result we want even when we require the model to fit observational constraints on all types of binary star, Algols, cataclysmic variables, X-ray binaries, symbiotics etc. Thus we stress that the results of binary population synthesis should not be glibly trusted.

\section{The Supernova Rate}

The observed rate of SNe Ia is $4 \pm 2$ per 1,000 yr per galaxy like our own (Cappellaro et al. 1997). Table 1 gives the rate for various possible progenitors for two of our population syntheses that differ only in the value of $\alpha_{\mathrm{CE}}$. We have chosen the primary mass $M_{1}$ from the mass function of Kroupa, Tout and Gilmore (1993), the secondary mass $M_{2}$ so as to give a uniform distribution of mass ratio $q=M_{2} / M_{1}$ and the semi-latera recta $l$ from a flat distribution in $\log l$ with $3<l / R_{\odot}<3 \times 10^{6}$. The binary fraction and galactic model are condensed into the statement that one binary system with $M_{1} \geq 0.8 M_{\odot}$ forms per year per galaxy, as is appropriate for our own (Hurley, Tout \& Pols 2002). 
The three major groups of progenitors can be further subdivided each into two distinct channels. The exploding Chandrasekhar-mass CO white dwarfs can be split into those that have dynamically unstable (coalescence) and those that have dynamically stable (accretion) mass transfer. The ELDs can be split into those $\mathrm{CO}$ white dwarfs accreting from a He white dwarf and those accreting from a naked helium-burning star. The exploding He white dwarfs, which we recall are not likely SNe Ia, can again be divided into those accreting from a white dwarf and those accreting from a naked helium star.

It is immediately apparent that by varying $\alpha_{\mathrm{CE}}$ alone we can fit the observed rate with whichever subset of the progenitors we please. Because of the two common-envelope phases involved the only other systems to be so strongly affected by $\alpha_{\mathrm{CE}}$ are the non-interacting double degenerate systems but their numbers are not yet sufficient to constrain $\alpha_{\mathrm{CE}}$ independently (Maxted \& Marsh 1999). The well-studied cataclysmic variables usually only experience a single common-envelope phase in their evolution and so their numbers, which are uncertain anyway, depend rather more weakly on $\alpha_{\mathrm{CE}}$. We reiterate that, even without varying the initial mass and period distributions, which would affect other types of binary, we are able to fit the SNe Ia with whichever subset of the progenitors we please. The problem is that there are too few observables to constrain the free parameters of the model.

\section{A Systematic Effect}

The Phillips relation between peak luminosity and light curve decay speed can be understood (Mazzali et al. 2001) because both the peak luminosity and the light curve speed depend on the amount of ${ }^{56} \mathrm{Ni}$ created in the thermonuclear runaway. The more ${ }^{56} \mathrm{Ni}$ produced, the brighter the peak while it is the line opacity of the iron peak elements that determines the speed of the light curve, the more iron the slower the light curve. Pinto \& Eastman (2001) explain the correlation in terms of more ${ }^{56} \mathrm{Ni}$ leading to a hotter, brighter supernova but one with a greater dispersion in the velocity of expanding ${ }^{56} \mathrm{Ni}$ layers, which delays the photons because they must escape a wider range of Doppler-shifted transitions. What is important to our argument is that the key parameter, controlling both peak luminosity and light curve speed, is the nickel mass. Thus if all the ${ }^{56} \mathrm{Ni}$ is produced in a single explosion we expect the correlation irrespective of the nature of the progenitor. However in ELDs ${ }^{56} \mathrm{Ni}$ is formed at two sites, the He envelope and then the CO core. Since the latter is still the most productive site it is still responsible for the peak but both sites provide opacity that slows the light curve decay.

Höflich, Wheeler \& Thielemann (1998) have pointed out that the precise composition of the $\mathrm{CO}$ core, particularly its carbon content, controls the peak 


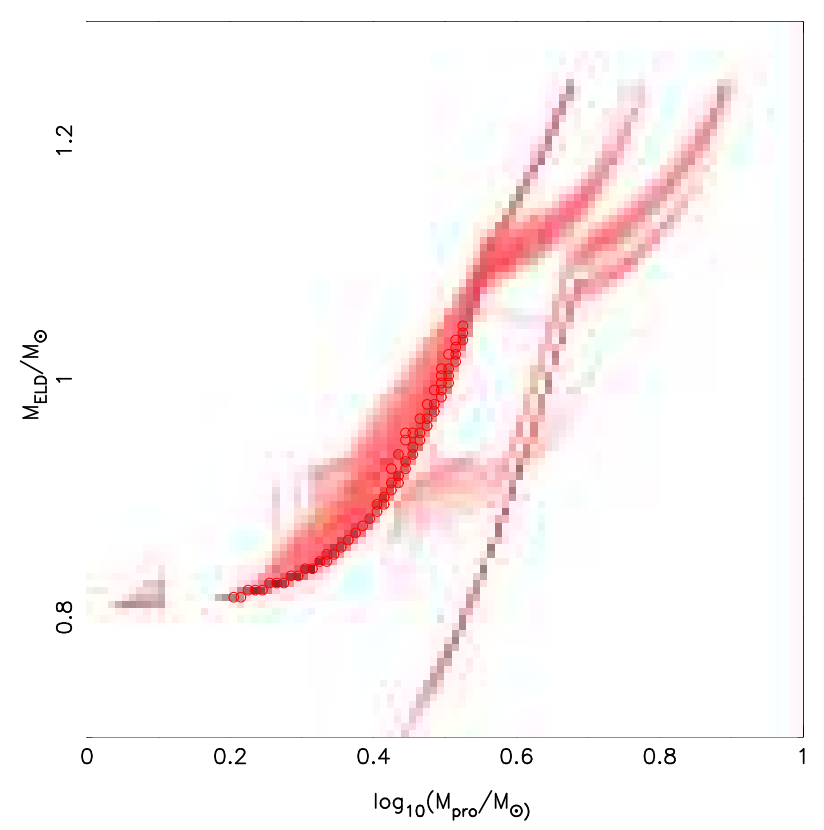

Fig. 1. The correlations between the total mass of ELDs (which predominantly determines light curve speed) and the mass of the progenitor of their CO cores (which predominantly determines the peak luminosity) evolved from stars with metallicity $Z=0.02$ and $\alpha_{\mathrm{CE}}=3.0$. The progenitor mass $M_{\text {pro }}$ is the initial main-sequence mass of the AGB star in which the CO core grew. It is this that determines the $\mathrm{C} / \mathrm{O}$ ratio. The grayscale is determined by the logarithm of the probability of SNe Ia falling at a particular point in this plane. The most common ELDs, picked out with circles, lie along the lower edge of the top-left band. The latter define a narrow (correlated) region so that we can hope to find correlations between properties of the supernovae for this metallicity. The rarer ELDs with naked helium star donors preferentially occupy the even narrower (more correlated) region to the lower right.

luminosity. The central composition depends on the initial, main-sequence mass of the asymptotic giant branch star that formed the $\mathrm{CO}$ white dwarf as its core (e.g. Umeda et al. 1999). It is established during the core helium burning phase before the star has expanded in radius on the AGB. If this mass were independent of the final ELD mass we could not hope to calibrate them locally. However, because more massive stars typically produce more massive remnants we expect some correlation between the mass of a $\mathrm{CO}$ white dwarf and that of its progenitor and so also between the mass of an ELD and its composition. The subtleties of binary star evolution mean that this is not a tight relation because the total mass of the $\mathrm{CO}$ core depends on when the AGB star interacts with its companion and so on the binary separation.

Fig. 1 is a plot of ELD mass against progenitor mass when $Z=0.02$ and $\alpha_{\mathrm{CE}}=$ 3.0. There is indeed a correlation with some scatter. Various less occupied regions come about because there are many routes by which stars can reach the final ELD. Apart from the difference in donor, the split between long initial period systems with two CE phases and short-period single-CE systems 


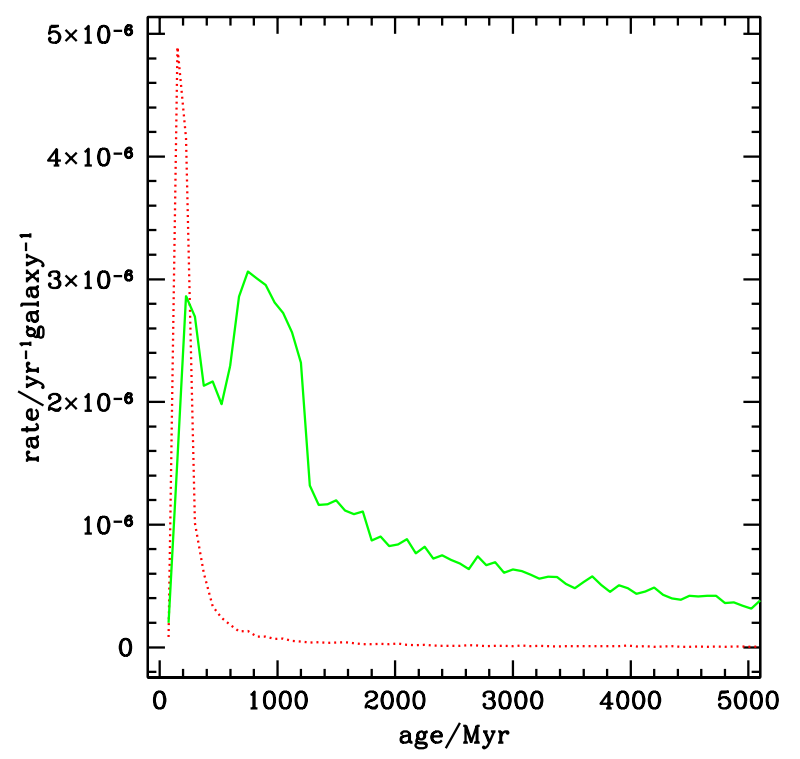

Fig. 2. Solid line - the contribution to the SNe Ia rate by ELDs at time $t$ from stars formed at $t$ - age. Dotted line - the equivalent contribution from merging pairs of $\mathrm{CO}$ white dwarfs. The ELD rate peaks less than $10^{9} \mathrm{yr}$ after the stars formed and the CO merger rate even sooner. The narrower ELD peak at this time comes from a subset of the ELDs with naked helium star donors whose CO components had massive progenitors $\left(5-8 M_{\odot}\right)$. It contains none of the helium white dwarf donors. These timescales are short compared with the cosmological age of the Universe at redshifts of $z=0$ and $z=1$, so that SNe Ia are essentially a product of the appropriate current generation of forming stars. In particular their metallicity is correlated with their redshift.

is most significant. Though the details are interesting they are not pertinent to this paper and we concentrate only on the highly occupied regions. Let us now suppose that the progenitor mass predominantly determines the peak luminosity while the total ELD mass predominately determines the light curve speed. The correlation between the two leads to the empirical relation used to calibrate the peak luminosities, and the scatter in this relation is due to the variations in formation pathway. If $\alpha_{\mathrm{CE}}=1$ the region containing the most common ELDs is squeezed to the upper left of this distribution and the gap just below $M_{\text {pro }}=10^{0.2}$ is filled in but the correlation still exists. In practice we expect both the peak luminosity and light curve shape to depend on some combination of both masses but our arguments are unchanged. There is still a one parameter family of SNe Ia. Now our population synthesis reveals that the peak rate of ELDs occurs about $10^{9} \mathrm{yr}$ after their progenitors formed (Fig. 2), just the time taken for intermediate mass stars to evolve off the main sequence. A similar conclusion can be reached by examination of Fig. 2 of Yungelson \& Livio (2000). Therefore local SNe Ia (even those in old galaxies with currently low star formation rates) have typically population I progenitors with a metallicity of $Z=0.02$. At a redshift of $z \approx 1$ the Universe was only 


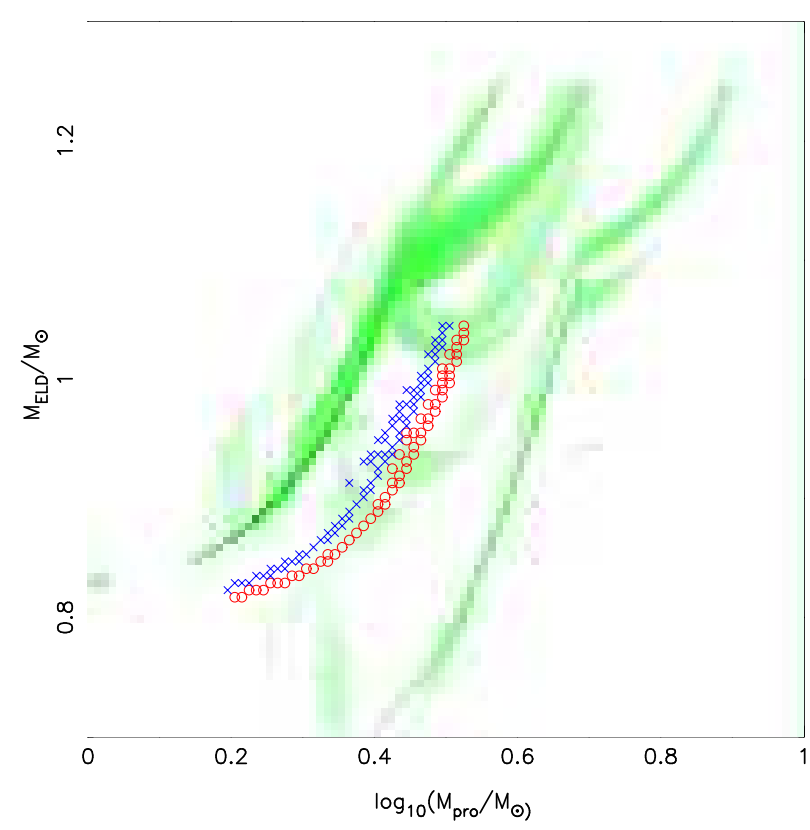

Fig. 3. Grayscale, as in Fig. 1, of the correlations for ELDs evolved from progenitors with $Z=0.001$. Open circles are the most common ELDs from progenitors of $Z=0.02$ (as in Fig. 1) while crosses are for the similarly most common ELDs from progenitors of $Z=0.01$. The systematic offset in these correlations leads to a systematic shift in the calibration of peak SN Ia luminosity by light curve shape. A given total ELD mass corresponds to a 30 per cent lower progenitor mass at $Z=0.001$ than at $Z=0.02$ so that the absolute brightness could be overestimated.

one third of its current age and the local metallicity was lower. Precisely how much lower is quite uncertain but our point is most clearly made by comparing a population II synthesis with $Z=0.001$ with that at $Z=0.02$. Fig. 3 compares the correlations between ELD and progenitor masses. There is a systematic shift in the correlation but the spread is little changed. As a result ELDs from population II progenitors could appear to have a very similar distribution of peak luminosities and light curve shapes to population I but at the same time have a systematic shift in the relation used to calibrate their peak luminosities. Indeed a similar total ELD mass at $Z=0.001$ is now mapped to a progenitor some 30 per cent less massive than at $Z=$ 0.02. Equivalently a certain speed of light curve decay at $Z=0.001$ could correspond to a significantly fainter peak luminosity than at $Z=0.02$. Though $Z=0.001$ is most likely an underestimate of the metallicity at a redshift of $z=1$, the effect is still apparent at $Z=0.01$, which is almost certainly an overestimate, (see Fig. 3).

The analysis by Kawai et al. (1987) of helium accreting spherically on to a CO white dwarf showed that, at accretion rates above $3 \times 10^{-8} M_{\odot} \mathrm{yr}^{-1}$, the helium burns non-degenerately as it accretes. Models we have made with the Eggleton stellar evolution code (Pols et al. 1995) confirm this and find degenerate ignition to be possible, even for spherical accretion, if accretion 
rates are less than $10^{-9} M_{\odot} \mathrm{yr}^{-1}$. Because of their larger size naked helium stars can feed CO white dwarfs at less than this rate. In Figs 1 and 3 these ELDs lie preferentially on the narrower correlation, at higher progenitor mass, to the right of the main cluster. This region does not appear to suffer the same systematic shift with metallicity. However, even at $Z=0.01$, the $\mathrm{C} / \mathrm{O}$ ratio is significantly higher than at $Z=0.02$. For instance models made with the Eggleton code (Pols et al. 1998) show that a $2.5 M_{\odot}$ progenitor with $Z=$ 0.01 produces a $\mathrm{CO}$ white dwarf with central carbon abundance $X_{\mathrm{C} 12}=0.15$ compared with 0.12 for a star of the same mass but $Z=0.02$. A similar abundance is shared by $\mathrm{CO}$ white dwarfs from $2.0 M_{\odot}$ progenitors when $Z=$ 0.02. These conclusions are based only on our simple arguments that should be tested with detailed explosion models before any firm deductions can be drawn either way. They are however sufficient to cast reasonable doubt on the fact that SNe Ia are standard candles independent of redshift, at least until we can be sure of a sample that is not contaminated with ELDs.

\section{$6 \quad$ Nucleosynthesis}

We might hope that if we include nucleosynthesis in population synthesis we can tap into many more observable quantities from stellar abundances of elements to meteoritic and terrestrial isotopic ratios. Unfortunately this is not without the expense of introducing new parameters to our model but we can reduce the ratio of parameters to observables. Of particular interest to us for supernovae are the iron group elements and heavier isotopes that are not synthesized in earlier phases of stellar evolution such as s-processing in AGB stars. The greatest uncertainty is introduced by our poor understanding of the mechanism by which SNe II explode. It is not certain how much of their iron core is trapped in or falls back on to the newly formed neutron star and how much is expelled to the ISM. The iron group production by the various core collapse supernovae, SNIb/c and SNII, is difficult to quantify because it critically depends on the poorly understood mechanism that drives the explosion. We can make an estimate based on the work of Woosley \& Weaver (1995). Their Table 5 shows a yield that varies from $0.04 M_{\odot}$, for a $12 M_{\odot}$ to $0.2 M_{\odot}$ for a $22 M_{\odot}$ star but for most masses the yield is below $0.1 M_{\odot}$. Above $25 M_{\odot}$ their yields vary greatly with their, imposed, piston velocity. If blackhole remnants are common the yields are very small. In any case these very massive progenitors ought to be much rarer. We use an average yield of about $0.08 M_{\odot}$ of Fe (mostly as ${ }^{56} \mathrm{Ni}$ ) expelled to the ISM per SN II as a reasonable but rather uncertain estimate. Because ${ }^{56} \mathrm{Ni}$ decay is the major source of energy in the SNe Ia their iron production is much better determined. Observations (Contardo, Leibundgut \& Vacca 2000) indicate a range in ${ }^{56} \mathrm{Ni}$ masses from $0.11 M_{\odot}$ for SN1991bg to $1.14 M_{\odot}$ for $\mathrm{SN} 1991 \mathrm{~T}$ with an average of $0.58 M_{\odot}$ 
for nine SNe Ia. For our purposes we would like to know the yield for each of our exploding white dwarfs and so we appeal theoretical models. Exploding Chandrasekhar-mass CO white dwarfs give about $0.63 M_{\odot}$ each (Thielemann, Nomoto \& Yokoi 1986). The ELD production depends on both CO core mass and He envelope mass (at least one extra parameter for the model) but the results of Livne and Arnett (1995) can be fitted quite well by

$$
M_{\mathrm{Ni}}=0.75 M_{\odot}-3.0\left(M_{\mathrm{CO}}-1.0 M_{\odot}\right)^{2} / M_{\odot}
$$

where $M_{\mathrm{Ni}}$ is the total mass of ${ }^{56} \mathrm{Ni}$, that will decay to ${ }^{56} \mathrm{Fe}$, produced by an ELD with $\mathrm{CO}$ core mass $M_{\mathrm{CO}}$. The mass of iron liberated by exploding $\mathrm{He}$ white dwarfs would again depend very much on the mass and accretion rate. An appropriate model (Woosley, Taam \& Weaver 1986) gives $0.45 M_{\odot}$ per explosion. We have included these yields in our population synthesis calculations and Table 1 lists the yields per 1,000 yr per galaxy for the model with $\alpha_{\mathrm{CE}}=3$. If an average SN II contributes about $0.08 M_{\odot}$ and their rate is about thrice that of SNe Ia (Cappellaro et al. 1997) then the iron contribution of SNe Ia is about two and a half times that of SNe II whatever the true progenitor.

\subsection{Explosive Helium Burning}

What might distinguish ELDs from exploding Chandrasekhar-mass CO white dwarfs? In the latter the thermonuclear runaway is confined to the $\mathrm{CO}$ rich mixture where it produces the ${ }^{56} \mathrm{Ni}$ and the Si peculiar to SNe Ia spectra. In the ELDs it occurs both in the CO core and in the He-rich envelope. In this envelope a small but very significant fraction of nuclei do not reach the end of the $\alpha$-burning chain but freezeout as the envelope expands. Of particular interest are the heavier isotopes such as ${ }^{44} \mathrm{Ti}$ and ${ }^{48} \mathrm{Cr}$ which are not readily produced elsewhere. We concentrate on ${ }^{44} \mathrm{Ti}$ that decays, via ${ }^{44} \mathrm{Sc}$, to ${ }^{44} \mathrm{Ca}$ in the ISM. The mass ratio of ${ }^{44} \mathrm{Ca} /{ }^{56} \mathrm{Fe}$ in the Solar System is $1.2 \times 10^{-3}$. An average SN II yields at most a ratio of about $6 \times 10^{-4}$ (Timmes et al. 1996) and an exploding Chandrasekhar-mass white dwarf only $3 \times 10^{-5}$ (Livne \& Arnett 1995). As Timmes et al. point out these two alone cannot account for the Solar-System abundance and we must turn to explosive He burning.

The model for the exploding He white dwarf that gave $0.45 M_{\odot}$ of iron (Woosley, Taam and Weaver 1986 ) yields $8.9 \times 10^{-3} M_{\odot}$ of ${ }^{44} \mathrm{Ti}$ so that only one such explosion per eighteen SNe II or six SNe Ia would be enough to account for the Solar-System ${ }^{44} \mathrm{Ca}$. We note however that our calculations with the Eggleton code show that it is very easy to raise the degeneracy of a He white dwarf by accretion before the triple- $\alpha$ reaction begins. The white dwarf becomes a naked helium burning star and subsequently a $\mathrm{CO}$ white dwarf without any explosive helium burning. In addition there are no obvious observed candi- 
dates for such explosions, which ought to be almost as bright as SNe Ia and we might reasonably discount them altogether.

The contribution from ELDs, not surprisingly, depends, like the Fe yield, on both the CO core mass and the He envelope mass. A fit to the models of Livne and Arnett (1995) is

$$
M_{\mathrm{Ti}}= \begin{cases}0.0033 M_{\odot}+0.143\left(M_{\mathrm{CO}}-0.8 M_{\odot}\right)^{2} / M_{\odot} & M_{\mathrm{CO}}<0.8 M_{\odot} \\ 0.0033 M_{\odot} & M_{\mathrm{CO}} \geq 0.8 M_{\odot}\end{cases}
$$

where $M_{\mathrm{Ti}}$ is the total mass of ${ }^{44} \mathrm{Ti}$, that will decay to ${ }^{44} \mathrm{Ca}$ produced by an ELD with $\mathrm{CO}$ core mass $M_{\mathrm{CO}}$. Again for the model with $\alpha_{\mathrm{CE}}=3$, the mass of ${ }^{44} \mathrm{Ti}$ returned to the ISM by each of the various progenitor types is recorded in Table 1 and the final column gives the Solar-System ratio if only that progenitor type is combined with thrice as many SNe II. ELDs give a ratio that is four times as large as is measured and so are unlikely to be the dominate type of SNe Ia. Exploding Chandrasekhar-mass CO white dwarfs give a ratio over six times too small. If we exclude the $\mathrm{CO}$ white dwarfs accreting from He white dwarfs on the grounds that they can burn helium non-degenerately then the combination of the ELDs accreting from naked helium stars, all the exploding Chandrasekhar-mass CO white dwarfs and the SNe II, with no exploding He white dwarfs, give a ratio of $2.0 \times 10^{-3}$ within a factor of two of the measured value. This is therefore our favoured model based on these calculations. The progenitors of 40 per cent of its SNe Ia are ELDs which are unlikely to be standard candles.

\section{Conclusions}

Though they are now quite unfashionable we have investigated whether or not edge-lit SNe Ia could behave as standard candles. We have so identified a systematic effect that could undermine the cosmological interpretation of SN Ia measurements at redshift $z \approx 1$ because it ensures that both nearby and distant SNe Ia appear to belong to a one parameter family that should obey a correlation between peak luminosity and light curve shape but at the same time hides a second parameter, metallicity. For this to be important a significant number of SNe Ia would need to be helium edge-lit detonations of CO white dwarfs. Unfortunately we find the results of binary population synthesis (such as Hurley et al. 2001) too unreliable to produce absolute numbers. There are just too many free parameters in the included physics and the initial distributions of binary properties. On the other hand the relative numbers used in identifying the systematic effects of metallicity on ELDs do not rely heavily on any of these many assumptions. If we include nucleosynthesis 
in population synthesis we find that 40 per cent of SNe Ia would need to be ELDs if they are the major source of ${ }^{44} \mathrm{Ca}$ in the solar system.

Any measurements of the metallicity of supernova progenitors can be used to test for such effects. Since we expect the majority of SNe Ia to result from relatively recent star formation (our Fig. 2 or Fig. 2 of Yungelson \& Livio 2000) measurement of the metallicity of intermediate and massive stars $\left(M \geq 2 M_{\odot}\right)$ in the host galaxy is also appropriate. Branch, Romanishin \& Baron (1995) found that the distribution of peak SNe Ia luminosities in redder galaxies has a higher dispersion, before correcting for light curve shape, than that of those in bluer galaxies. This is consistent with SNe Ia in an older red galaxy coming from a wider range of progenitor metallicities but is otherwise inconclusive. Further investigations of how SNe Ia might vary with galaxy type are underway (e.g. Schmidt, private communication) and we eagerly await the results.

We conclude that we find no evidence that the majority of SNe Ia should not be standard candles obeying a one parameter Phillips relation. However if any can be identified as ELDs they should be used neither to establish the Phillips relation nor in the distant samples. In particular any object that shows similarities to SN1991T should be excluded.

\section{ACKNOWLEDGMENTS}

We thank Zhanwen Han, Andrew King, Robert Kirshner, Paolo Mazzali, Philipp Podsiadlowski, James Pringle and Brian Schmidt for stimulating conversations and helpful comments. ER and CAT thank the Australian National University and its Astrophysical Theory Centre for hospitality and generous support. CAT is very grateful for an advanced fellowship from PPARC and to Churchill College for a Lectureship in Mathematics. DW is grateful to the UK PPARC for a visiting fellowship at the IOA, Cambridge. We also thank the various referees who have taken the time to read this work in detail and provided a wide range of criticisms that have, we hope, improved our presentation.

\section{References}

[1] de Bernardis P. et al., 2000, Nat, 404, 955

[2] Branch D., 1998, ARA\&A, 36, 17

[3] Branch D., 2000, in Young Supernova Remnants, eds. S. Holt, U. Hwang, ASP Conf. Ser. 565, 31 
[4] Branch D., Nomoto K., 1986, A\&A, 164, L13

[5] Branch D., Romanishin W., Baron E., 1996, ApJ, 465, 73

[6] Cappellaro E., Turatto M., Tsvetkov D. Y., Bartunov O. S., Pollas C., Evans R., Hamuy M., 1997, A\&A, 322, 431

[7] Contardo G., Leibundgut B., Vacca W. D., 2000, A\&A, 359, 876

[8] Cumming R. J., Lundquist P., Smith L. J., Pettini M., King D. L., 1996, MNRAS, 283,1355

[9] Eck C. R., Cowan J. J., Roberts D. A., Boffi F. R., Branch D., 1995, ApJ, 451, L53

[10] Filippenko A. V. et al., 1992, AJ, 104, 1543

[11] Fisher A., Branch D., Hatano K., Baron E., 1999, MNRAS 304, 67

[12] Hachisu I., Kato M., Nomoto K., 1996, ApJ, 470, L97

[13] Hillebrandt W., Niemeyer J.C., 2000, ARA\&A, 38, 191

[14] Höflich P., Khokhlov A., 1996, ApJ, 457, 500

[15] Höflich P., Wheeler J. C., Thielemann F. K., 1998, ApJ, 495, 617

[16] Hurley J. R., Tout C. A., Pols O. R., 2002, MNRAS, 329, 897

[17] Kahabka P., van den Heuvel E. P. J., 1997, ARA\&A, 35, 69

[18] Kawai Y., Saio H., Nomoto K., 1987, ApJ, 315, 229

[19] Kroupa P., Tout C. A., Gilmore G., 1993, MNRAS, 262, 545

[20] Liu W., Jeffery D. J., Schultz D. R., 1997, ApJ, 486, L35

[21] Livne E., Arnett D., 1995, APJ, 452, 62

[22] Livne E., Glasner A. S., 1990, ApJ, 361, 244

[23] Maxted P. F. L., Marsh T. R., 1999, MNRAS, 307, 122

[24] Mazzali P. A., Lucy L. B., 1998, MNRAS, 295, 428

[25] Mazzali P. A., Nomoto K., Cappellaro E., Nakamura T., Umeda H., Iwamoto K., 2001, ApJ, 547, 988

[26] Nomoto K., Iben I. Jr., 1985, ApJ, 297, 531

[27] Nugent P., Baron E., Branch D., Fisher A., Hauschildt P. H., 1997, ApJ, 485, 500

[28] Paczyński B., Żytkow A. N., 1978, ApJ, 222, 604

[29] Perlmutter S. et al., 1999, ApJ, 517, 565 
[30] Phillips M. M., 1993, ApJ, 413, L105

[31] Pillips M. M., Lira P., Suntzeff N. B., Schommer R. A., Hamuy M., Maza J., 1999, AJ, 118, 1766

[32] Pinto P. A., Eastman R. G., 2001, New ast., 6, 307

[33] Pols O. R., Schröder K-S., Hurley J. R., Tout C. A., Eggleton P. P., 1998, MNRAS, 298, 525

[34] Pols O. R., Tout C. A., Eggleton P. P., Han, Z., 1995, MNRAS, 274, 964

[35] Regös E., Tout C. A., 1995, MNRAS, 273, 146

[36] Riess A. G. et al., 1998, AJ, 116, 1009

[37] Rowan-Robinson M., 2002, MNRAS, 332, 352

[38] Thielemann F.-K., Nomoto K., Yokoi K., 1986, A\&A, 158, 17

[39] Timmes F. X., Woosley S. E., Hartmann D. H., Hoffman R. D., 1996, APJ, 464,332

[40] Umeda H., Nomoto K., Kobayashi C., Hachisu I., Kato M. 1999, ApJ, 522, L43

[41] Warner B., 1995, Cataclysmic Variable Stars. Cambridge Univ. Press, Cambridge

[42] Wittman D. M., Tyson J. A., Kirkman D., Dell'Antonio I., Bernstein G., 2000, Nat, 405, 143

[43] Woosley S. E., Taam R. E., Weaver T. A., 1986, ApJ, 301, 601

[44] Woosley S. E., Weaver T. A., 1994, ApJ, 423, 371

[45] Woosley S. E., Weaver T. A., 1995, ApJS, 101, 181

[46] Yungelson L. R., Livio M., 2000, ApJ, 528, 108 